\title{
DEVELOPMENT OF AN ALL-FIBER COHERENT DOPPLER LIDAR IN THE IAO SB RAS \\ Artem Sherstobitov ${ }^{1,2 *}$, Viktor Banakh ${ }^{1}$, Alexander Nadeev ${ }^{1}$, Igor Razenkov ${ }^{1}$, Igor Smalikho ${ }^{1}$, Andrey Falits ${ }^{1}$
}

${ }^{1}$ Institute of Atmospheric Optics of Siberian Branch of the Russian Academy of Sciences, Russia, ${ }^{2}$ National Research Tomsk State University, Russia, *shrarm@iao.ru

\begin{abstract}
Paper presents a model of the all-fiber pulsed coherent Doppler lidar (IAO-lidar) build in the IAO SB RAS. Here is described lidar design, the algorithm for processing of lidar signals and the software-hardware system that implements signal processing in real time, created with the use of open source software. The results of joint measurements of the radial velocity by the IAOlidar and the HALO Photonics (Stream Line) lidar are given.
\end{abstract}

\section{INTRODUCTION}

For experimental study of turbulent wind fields of the atmospheric boundary layer and coherent structures, including aircraft wake vortex, a pulsed coherent Doppler lidar (IAO-lidar) was recently build at the Institute of Atmospheric Optics (IAO) of the SB RAS. This paper presents design features of the IAO-lidar, algorithm for processing lidar signals, its implementation in real time, and comparative measurements of the radial velocity by IAO-lidar and by HALO Photonics (Stream Line) lidar.

\section{LIDAR DESIGN}

A construction of the developed IAO-lidar is shown in Figure 1. CW laser radiation $(20 \mathrm{~mW}$, $1550 \mathrm{~nm}$ ) with a frequency $f_{0}$ passes through coupler $75 / 25$, enters the isolator $(75 \%)$, and then goes to the circulator. After the circulator there is an acoustic optical modulator that generates the sounding pulses. At the other end of the modulator we have the Bragg grating (FBG), which acts as a selective mirror. Radiation passes through the modulator twice. Double passage of radiation allows suppress the signal leakage after the sounding pulse. This is the basic difference between our design and the well-known [1]. The frequency of the radiation after the modulator is increased by $f_{i}=80 \mathrm{MHz}$. The optical pulse after double passage through the modulator returned to the circulator and redirected to an erbium amplifier (EDFA). Second circulator is combined with EDFA in one module.

Sounding pulse with the frequency $f_{0}+f_{i}$ goes to the atmosphere via telescope. The backscattered signal coming from the atmosphere due to Doppler effect acquires an additional frequency shift $f_{d}$, depending from the radial component of the wind speed. Two inputs of the coupler 50/50 receive $\mathrm{CW}$ laser signal with frequency $f_{0}$ and continuous echo from the atmosphere with frequency $f_{0}+f_{i}+f_{d}$. Coupler divides CW signal and return in half, and then both go to the two inputs of the balanced PIN detector. Balanced detector extracts $f_{i}+f_{d}$ frequency component. The pulse repetition frequency is $10 \mathrm{kHz}$, the average outgoing power is $400 \mathrm{~mW}$. Aperture of the transceiver telescope is $50 \mathrm{~mm}$. The system is equipped with the scanner.

The fiber junctions in Figure 1 are shown with an inclined short lines. All fibers maintaining radiation polarization (PM). To prevent reflections at the joints, the type of connectors FC/APC is used.

\section{LIDAR SINGNAL PROCESSING ALGORITHM}

The radial velocity is calculated from the Doppler frequency shift of the signal $X(t)$ from the balanced PIN detector. Signals $X(t)$ are digitized by ADC, then from the signal array $X_{l}\left(n t_{s}\right)$ the signal power spectra $S_{m}\left(f_{k}\right)$ is calculated by the 
use of FFT with the averaging over $L$ sounding pulses:

$$
S_{m}\left(f_{k}\right)=\frac{t_{S}}{L N T_{W}} \sum_{l=1}^{L}\left|S_{m l}\left(f_{k}\right)\right|^{2}
$$

where

$$
S_{m l}\left(f_{k}\right)=\sum_{n=-N / 2}^{N / 2-1} X_{l}\left(n t_{s}+2 R_{m} / c\right) \exp \left(-\frac{2 \pi j n k}{N}\right) W(n),
$$

$t_{s}$ is the ADC sampling rate, $f_{k}=k \Delta f$, $\Delta f=\left(N t_{s}\right)^{-1}, \quad k=0,1,2, \ldots, N / 2, S_{m}\left(f_{k}\right)$ is the spectrum at the range $R_{m}=R_{0}+m \Delta R$, $m=0,1,2, \ldots, M$ is the number of the power spectrum, $\Delta R$ is the range gate length, $W(n)$ is the window function, $T_{W}$ is the effective width of the window function, $c$ is light speed.

The following parameters were used: $t_{s}=4 \mathrm{~ns}$; the dimension of the Fourier transform $N=64$, which corresponds to the spectral resolution $\Delta f=3.9$ MHz; $M=390$; a rectangular time window was used, that is, $W(n)=1$; for more accurate calculation of the radial velocity profile from the power spectra Fourier interpolation was used.

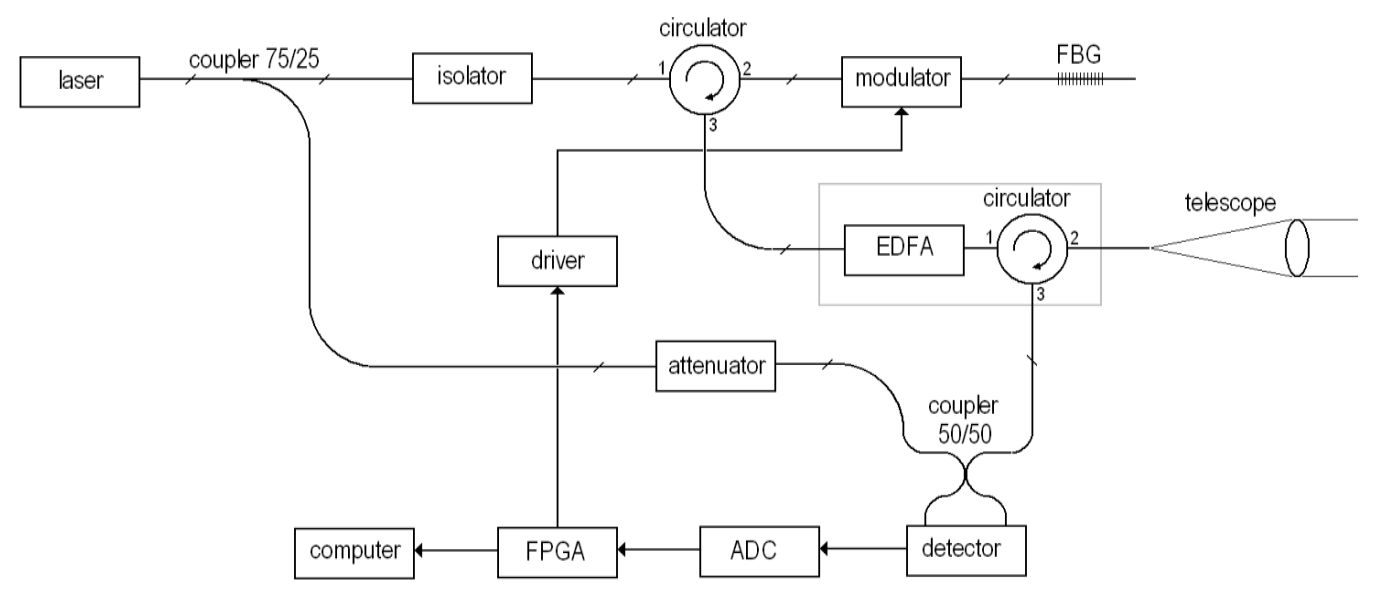

Fig. 1. Diagram of the all-fiber Doppler IAO-Lidar.

In the general case, the power spectrum $S(f)$ of a lidar signal can be represented as the sum of the signal $S_{S}(f)$ and noise $S_{N}(f)$ components [2]. To eliminate the influence of the noise component, two methods were applied: a) heuristic calibration method: the power spectra sequence obtained by subtraction of the blocked beam sequence from spectrum $S(f)$;

b) noise subtraction: this method is described in [2], that is, the spectrum $S_{m}(f)$ starting from the index $M_{1}$ is considered as the noise, thus, the average value of the noise spectrum is calculated by

$$
<S_{N}(f)>=\sum_{m=M_{1}}^{M-1} S_{m}(f) /\left(M-M_{1}\right)
$$

Signal-to-noise ratio (SNR) was estimated as follows:

$$
\mathrm{SNR}_{m}=\frac{\sum_{k=b_{1}}^{b_{2}}\left(S_{m}\left(f_{k}\right)-<S_{N}\left(f_{k}\right)>\right)}{\sum_{k=b_{1}}^{b_{2}}<S_{N}\left(f_{k}\right)>},
$$

where $b_{1}=\left(f_{i}-B / 2\right) / \Delta f, \quad b_{2}=\left(f_{i}+B / 2\right) / \Delta f$ and $B=50 \mathrm{MHz}$ is the bandwidth for estimation of the radial velocity.

\section{HARDWARE-SOFTWARE IMPLEMENTETION OF THE REAL TIME SIGNAL PROCESSING}

The hardware-software module consists of analogto-digital converter ADS4249EVM, TSW1400EVM board with field-programmable gate array (FPGA) STRATIX IV on it, and portable PC. 
The FPGA firmware calculates averaged power spectra by the formula (1) in real time, and controls the sounding pulse (Figure 2). The calculation of averaged power spectra is a conveyor algorithm using the open Fourier conveyor transform module [3] (Figure 3). The firmware is written in the Verilog HDL in the Intel Altera Quartus II development environment.

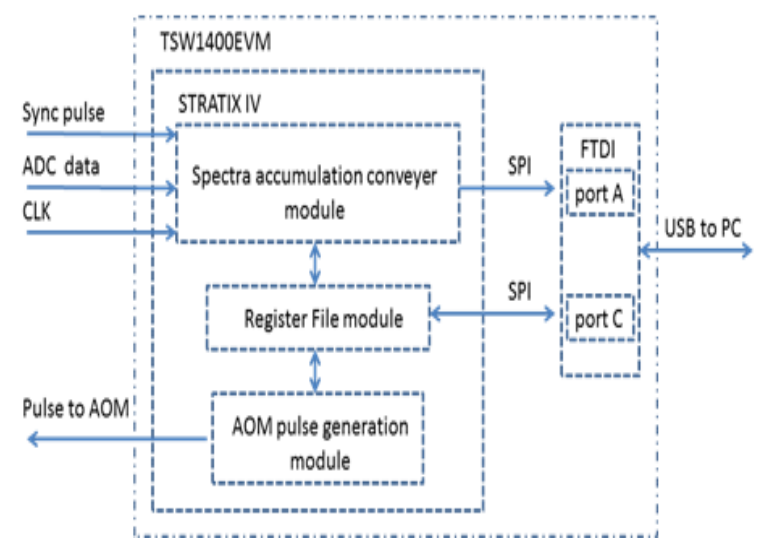

Fig.2. Software-hardware data processing system block diagram.

The PC program communicates with the FPGA via USB cable, connected to the FPGA board by using the 2 channels of FTDI chip, available on the TSW1400EVM. One channel are used for reading accumulated spectra (1) via SPI mode 3, another channel is also configured in SPI mode 3 and is used to read and write the block of registers to the FPGA.

The registers block is implemented as Verilog HDL module and is used for storing parameters ( $M, N$ in eq. 2, the time between the sounding pulses, etc.) Information about the ending current averaging by $L$ sounding pulses is also recorded to the register file.

Real-time spectra-accumulating conveyer shown at figure 3 uses floating point ( single precision float) based on open source Fourier conveyor transform module [3]. Floating point computations are quite slow, so we process four numbers of coming ADC samples per time. Parallel computations allow to reduce conveyer frequency from $250 \mathrm{MHz}$ ( ADC data-capturing frequency) to $62.5 \mathrm{MHz}$.
The PC program receives the average Doppler spectra as the input, from which the radial wind speed is calculated using the Doppler equation after Fourier interpolation of the spectra $S_{m}\left(f_{k}\right)$. The signal-to-noise ratio is calculated by Eq. (3).

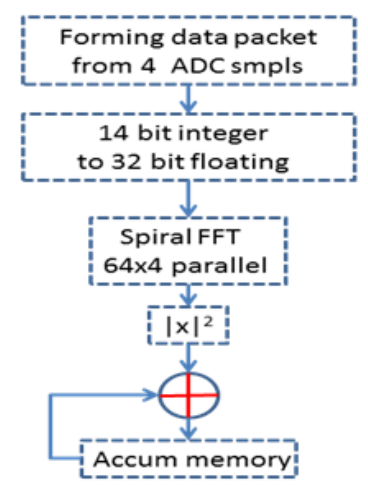

Fig.3. Spectra accumulating conveyer block, which implements equation (1) in real time.

\section{EXPERIMENT}

We carried out joint measurements of SNR and wind velocity by the IAO-lidar and the commercial lidar "Stream Line" made by HALO Photonics. Sounding of the radial velocity was held on fixed horizontal path (Fig. 4). Both paths were located in parallel at a distance of $\sim 3$ meters from each other. The results of sounding are presented at figures 5, 6. Figure 5 shows the range profiles of the signal-to-noise ratio. SNR curves point out that lidars characteristics are the similar. The sensing range is limited by $2 \mathrm{~km}$.

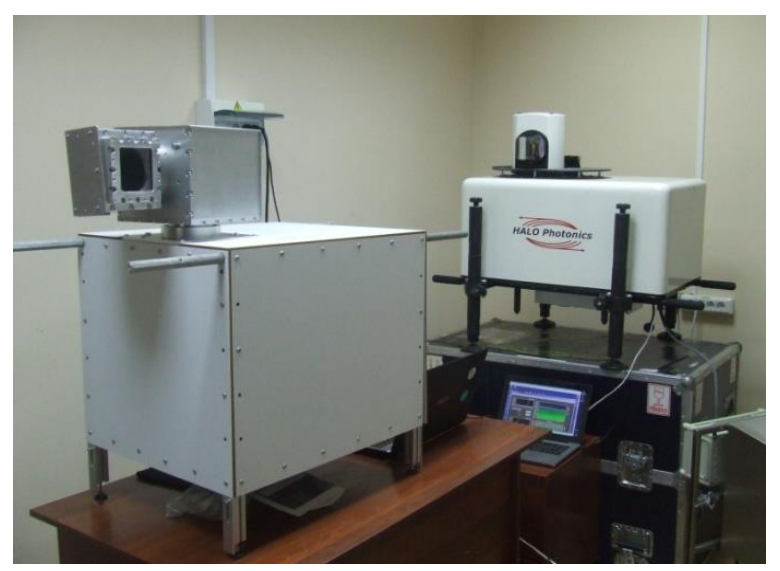

Fig. 4. Lidars: IAO -lidar (left) and "Stream Line" (right) during joint experiment. 


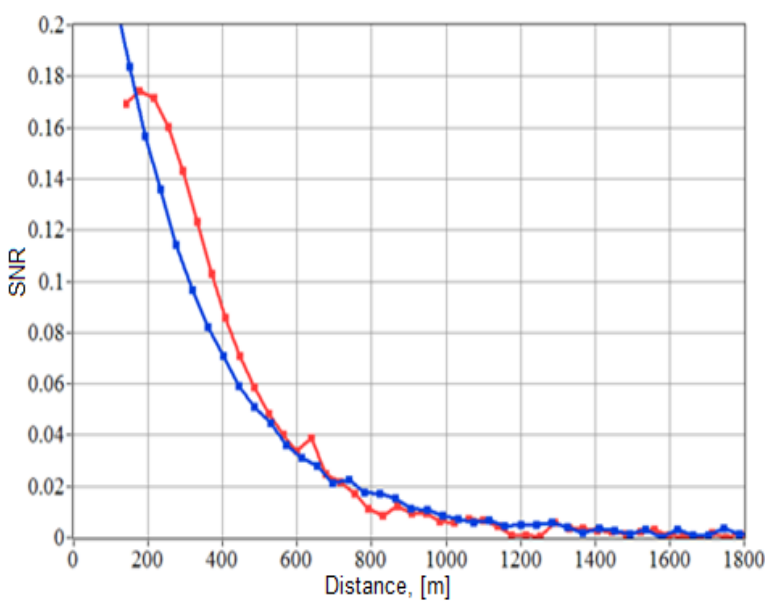

Fig.5. SNR measured by Stream Line lidar (blue line) and by IAO-lidar (red line) 04.02.2019.

The time dependence of the radial velocity measured by the IAO-lidar (Fig. 6) is similar to the results obtained from the Stream Line lidar data. The correlation coefficient between two curves is 0.84 .

\section{CONCLUSIONS}

Laboratory coherent Doppler all-fibered lidar was built at the IAO SB RAS for field measurements during atmospheric turbulence research experiments. The transmitter design is differ from the known ones by the additional fiber circulator and FBG, which provide a double pass of radiation through the modulator. This allows one to suppress the signal leakage after the sounding pulse and increase the signal-to-noise ratio.

Software-hardware module performs signal processing in real time. Moreover, the module was created using open source software, which made the module more portable and flexible. The field experiment was carried out together with the Stream Line lidar. In a result we have good correlation between measured lidar's wind radial velocities.

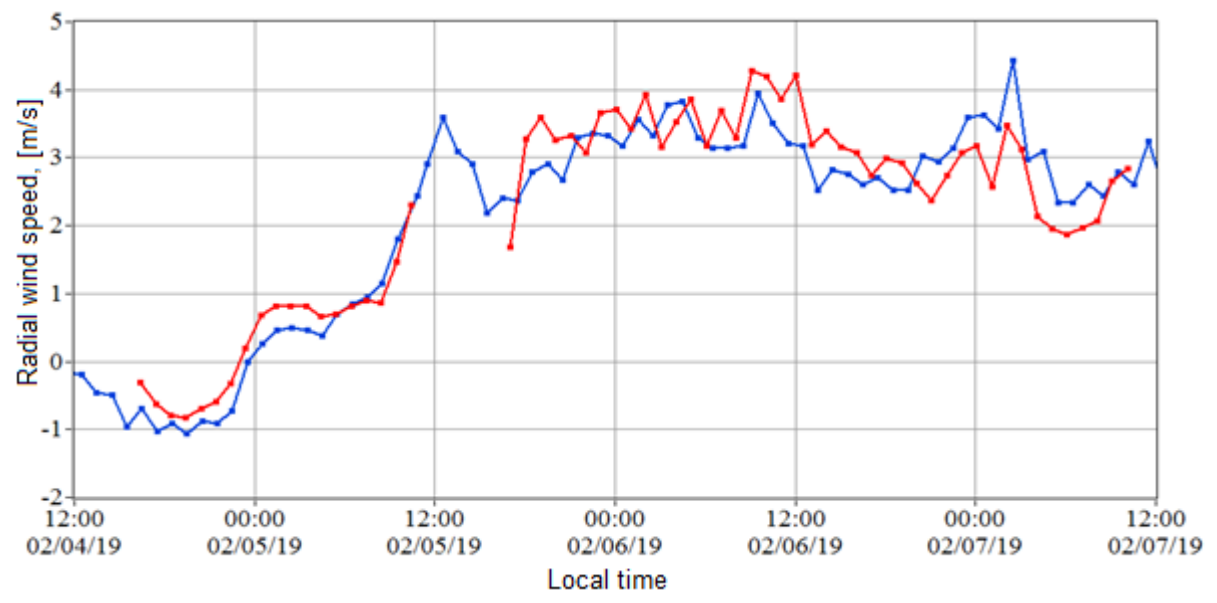

Fig.6. Time dependencies of radial wind speed at the distance of $442 \mathrm{~m}$ from lidars. Red line - IAO-lidar, blue line "Stream Line" lidar. Average time is one hour.

\section{REFERENCES}

[1] S. Kameyama, T. Ando, K. Asaka, Y. Hirano, and S. Wadaka "Compact all-fiber pulsed coherent Doppler lidar system for wind sensing", Applied Optics, 46, 1953-1962 (2007)

[2] Banakh V.A., Smalikho I.N. Coherent Doppler wind lidars in a turbulent atmosphere. Boston London: Artech House, 2013. 248 p.
[3] Verilog HDL generator of conveyer Fast Fourier transform: [link]. URL http://www.spiral.net/hardware/dftgen.html 